\title{
Polymer-Stabilized Micelles Reduce the Drug Rapid Clearance In Vivo
}

\author{
Shan-Ni Wen $\mathbb{D}^{1},{ }^{1}$ Chih-Hang Chu, ${ }^{2}$ Yu-Chao Wang $\mathbb{D}^{1},{ }^{1}$ Hsin-Ying Huang, ${ }^{1}$ Yu-Jing Wang, \\ Jia-Yi Lin, ${ }^{1}$ Hsiao-Ting Lu $\mathbb{D}^{1},{ }^{1}$ Sin-Jie Wang, ${ }^{1}$ and Chung-Shi Yang $\mathbb{D}^{1}$ \\ ${ }^{1}$ Institute of Biomedical Engineering and Nanomedicine, National Health Research Institutes, Zhunan, Miaoli 35053, Taiwan \\ ${ }^{2}$ Department of Applied Chemistry, National Chi Nan University, Puli, Nantou 54561, Taiwan \\ Correspondence should be addressed to Yu-Chao Wang; wyc@nhri.org.tw and Chung-Shi Yang; cyang@nhri.org.tw
}

Received 20 November 2017; Accepted 2 April 2018; Published 22 May 2018

Academic Editor: Stefano Bellucci

Copyright (C) 2018 Shan-Ni Wen et al. This is an open access article distributed under the Creative Commons Attribution License, which permits unrestricted use, distribution, and reproduction in any medium, provided the original work is properly cited.

\begin{abstract}
Micelles are self-assembled nanoscaled aggregates from amphiphilic unimers and can be used to encapsulate hydrophobic drugs. However, the dynamic exchanging of unimers between micelles and bulk solution often leads to micelle destabilization and subsequent leaking of the encapsulated substances. Thus, we incorporated a hydrophobic polymer into the micellar core for interlacing the unimers and stabilizing the micelle structure. The polymer-stabilized and non-polymer-stabilized micelles have similar physicochemical properties including small sizes $(\sim 35 \mathrm{~nm})$, negative surface charges $(\sim-35 \mathrm{mV})$, and high drug contents $(\sim 15 \%)$. Drugs encapsulated in polymer-stabilized micelles are released in a slower rate than are non-polymer-stabilized micelles. From in vivo pharmacokinetic studies, drugs loaded in polymer-stabilized micelles have lower clearance and higher plasma concentration and lower volume distribution than non-polymer-stabilized micelles have. In conclusion, polymerstabilized micelles can reduce rapid drug clearance via strengthening of the micellar structure and increase in the available drug amount in plasma, thus broadening pharmaceutical applications of micelles.
\end{abstract}

\section{Introduction}

Nanotechnology is widely employed in drug delivery systems (DDSs) $[1,2]$. In order to improve drug availability and to overcome the barriers of the biological system, the DDSs are generally designed to have sizes falling within the range of $1-100 \mathrm{~nm}$ [3]. In addition, other preferred properties of the DDSs would include biodegradability, biocompatibility, high loading capacity, good stability, high content of the drug in a final preparation, prolonged circulation, and ability to accumulate in required areas [4-6]. Common types of DDS include liposomes $[7,8]$, micelles [9-11], and polymeric nanoparticles [12-14].

PEG-PE micelles prepared from conjugates of polyethylene glycol (PEG) and phosphatidylethanolamine (PE) have a core-shell structure which can encapsulate hydrophobic drugs such as paclitaxel, parthenolide, tamoxifen, campotheicin, and budesonide [11]. Steric PEG block chains on the surface of micelles can decrease the opsonin protein binding in vivo and therefore block the capture by the reticuloendothelial system (RES) efficiently [15]. The low critical micellization concentration (CMC) value of PEG-PE compounds (in a $10^{-5}$ to $10^{-6} \mathrm{M}$ range) indicates that PEG-PE micelles may maintain their integrity even upon strong dilution (e.g., by the large blood volume upon intravenous administration) [9]. PEG-PE micelle sizes can be adjusted from $7 \mathrm{~nm}$ to $40 \mathrm{~nm}$ by altering PEG with different lengths [16]. These aforementioned small size and PEGylated surface make PEG-PE micelles able to accumulate in subcutaneous tumour [17] or the infract zone [18]. The PEG-PE micelles can be further modified with a functional group or antibody moiety on the surface to enhance their targeting efficiency [19]. PEG-PE micelles possess advantages such as small size, high solubility for hydrophobic drugs, low CMC, sustained release of drugs, targeting delivery, and potential for sterilization by filtration [20] for being a promising drug carrier [11].

However, this type of micelles is dynamic and in equilibrium with individual unimers that constantly undergo 


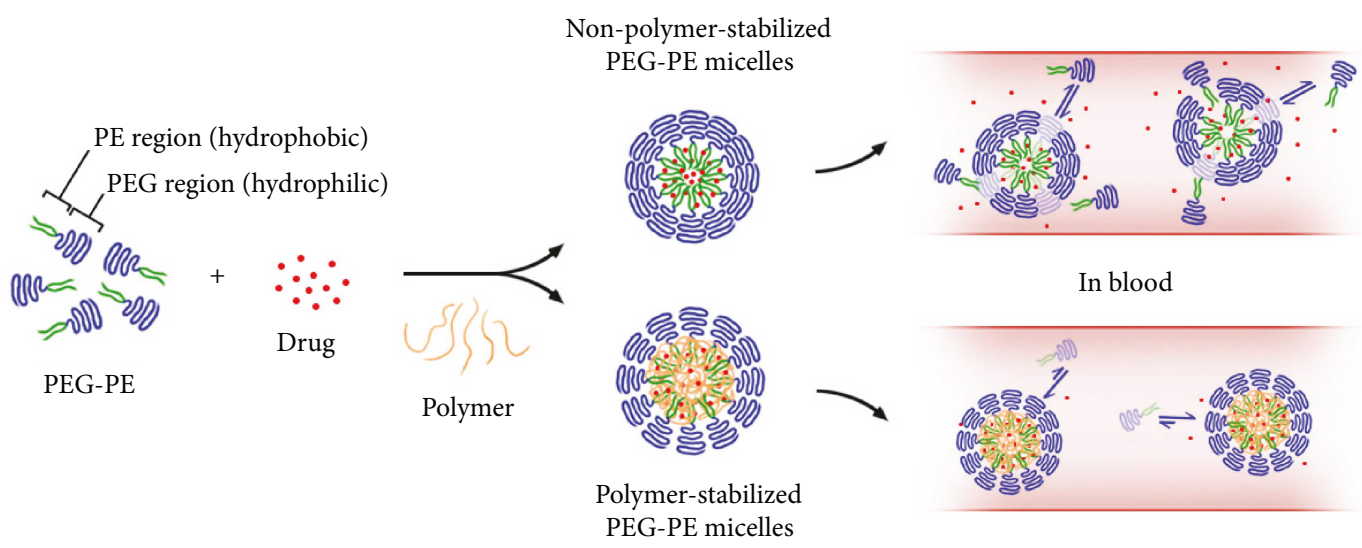

FIGURE 1: Scheme of polymer-stabilized PEG-PE micelles and nonpolymer-stabilized PEG-PE micelles. The polymer incorporation may interlace the PEG-PE unimers and reduce the dynamic exchange of the unimers and therefore enhance the micelle stability in vitro and in vivo.

exchange between the bulk and the micelles [21, 22]. The entrapped drugs could leak out while micelles repeatedly disintegrate and reassemble [23]. Such drug leaking issue will limit its pharmaceutical applications. Therefore, we designed a polymer-stabilized PEG-PE micelle which can maintain the micelle integrity and reduce the drug leakage. PLGA (poly(lactic-co-glycolic acid)), a hydrophobic biodegradable polymer, was selected to be incorporated into the inner core of the micelles to interlace the carbon chains of PEG-PE unimers and was presumed to decrease unimer disassembly which may stabilize the PEG-PE micelles. A schematic diagram is illustrated in Figure 1. The polymerstabilized PEG-PE micelles should have better stability than non-polymer-stabilized PEG-PE micelles, and they can hold entrapped drug molecules inside for longer periods. Since usually hydrophobic drug release from micelles will be cleared rapidly, such design can provide more available and accessible drugs in vivo.

Clarithromycin (CL) is a clinically used macrolide antibiotic and is selected as a model drug to be loaded into the PLGA-incorporated PEG-PE micelles (referred to as P-MCL) and the non-PLGA-incorporated PEG-PE micelles (referred to as MCL). All the physicochemical characterization, release profile in vitro, and pharmacokinetics in vivo of these two types of micelles were performed and analysed to examine whether polymer-incorporated micelles could act as a better drug carrier than non-polymerincorporated micelles.

\section{Materials and Methods}

2.1. Materials. Clarithromycin (CL), roxithromycin (ROX), poly(D,L-lactic-co-glycolic acid) (PLGA, lactide: glycolide $=50: 50$, MW $40 \mathrm{kD}-75 \mathrm{kD}$ ), ammonium acetate, potassium dihydrogen phosphate, phosphoric acid, phosphatebuffered saline (PBS), xylazine, and formic acid were purchased from Sigma-Aldrich (Saint Louis, MO, USA) and used as supplied. 1,2-Distearoyl-sn-glycero-3-phosphoethanolamine- $\mathrm{N}$-[methoxy(polyethylene glycol)-2000] (PEGPE) was purchased from Avanti Polar Lipids (Alabaster, AL, USA). Fetal bovine serum (FBS) was purchased from Thermo Fisher Scientific (Waltham, MA, USA). Zoletil was purchased from Virbac (Carros, France). Oasis WCX $\mu$ Elution SPE plates were products of Waters (Milford, MA, USA). Acetonitrile, chloroform, and methanol were HPLCgrade products of Honeywell International (Morristown, NJ, USA).

2.2. Preparation of $P-M C L$ and $M C L$. P-MCL was prepared by an oil-in-water emulsion solvent evaporation method similar to that reported previously [24]. Briefly, $7.5 \mathrm{mg}$ of $\mathrm{CL}, 10 \mathrm{mg}$ of PLGA, and $20 \mathrm{mg}$ of PEG-PE were dissolved in $1 \mathrm{~mL}$ of chloroform and was added into $3 \mathrm{~mL}$ of $\mathrm{H}_{2} \mathrm{O}$ with moderate stirring. The mixed solution was emulsified by sonication over an ice bath using a probe sonicator (XL-2000, Misonix, NY, USA) at $8 \mathrm{~W}$ output for 60 seconds. Chloroform was then evaporated from the colloidal suspension by a rotary evaporator. The suspension was centrifuged at $14000 \times \mathrm{g}$ for 30 minutes to discard the undesired big aggregates. The supernatant was collected and washed three times using an Amicon Ultra-4 centrifugal filter $(30 \mathrm{kD}$ MWCO, Merck Millipore, Billerica, MA, USA). For the preparation of MCL, the procedures were the same as in P-MCL, except that no PLGA was mixed in the first step.

2.3. Optimization of PLGA Incorporation. To determine the optimal PLGA incorporation amount for stabilizing micelles, P-MCLs with different feeding ratios of PLGA/PEG-PE $(0.25,0.5$, and 1$)$ were prepared. These P-MCLs were then incubated in $10 \%$ FBS with mild shaking at $37^{\circ} \mathrm{C}$ for the stability test. At $0,17,24,41$, and 65 hours, the size of each P-MCL was measured by DLS (dynamic light scattering) analysis using 90Plus Particle Size Analyzer (Brookhaven Instruments Corp., Holtsville, NY, USA).

2.4. Transmission Electron Microscopy (TEM) Imaging. TEM was performed on a Japan Hitachi H-7650 instrument with an acceleration voltage of $80-120 \mathrm{kV}$. TEM samples were prepared by administering the micelle suspension onto a copper grid followed by negative staining for 10 seconds at room temperature with freshly prepared and sterile-filtered $2 \%(w / v)$ uranyl acetate aqueous solution. 
2.5. Zeta Potential Measurements. Zeta potential was performed by a ZetaPALS analyser (Brookhaven Instruments Corp., Holtsville, NY, USA) with a $35 \mathrm{~mW}$ diode laser (660 $\mathrm{nm}$ in wavelength) and a detector with a scattering angle of 90 degrees. The measured results were analysed by a PALS zeta potential analyser (Brookhaven Instruments Corp., Holtsville, NY, USA).

2.6. Determination of Encapsulation Efficiency and Loading Content of CL. CL encapsulation efficiency (EE) was determined by applying (1), where $M_{\text {encapsulated }}$ is the mass of CL entrapped within the micelles and $M_{\text {add }}$ is the mass of the $\mathrm{CL}$ added in the preparation of micelles.

$$
\mathrm{EE}(\%)=\frac{M_{\text {encapsulated }}}{M_{\text {add }}} \times 100 \% \text {. }
$$

The CL loading content is defined as the mass fraction of CL in the micelles. The lyophilized micelles were weighted and then dissolved in 50\% acetonitrile solution, and CL was quantified by an Agilent (Santa Clara, CA, USA) highperformance liquid chromatograph (HPLC) equipped with a $\mathrm{C}_{18}$ column $(250 \mathrm{~mm} \times 4.6 \mathrm{~mm}$ id, $5 \mu \mathrm{m})$ at an absorption wavelength of $205 \mathrm{~nm}$. The mobile phase was composed of $50 \mathrm{mM}$ potassium dihydrogen phosphate ( $\mathrm{pH} 7.0)$ : acetonit rile $=60: 40(v / v)$ with a flow rate of $1.0 \mathrm{~mL} / \mathrm{min}$.

2.7. CL In Vitro Release Profiles. $1 \mathrm{mg}$ of micelles suspended in $1 \mathrm{~mL}$ of phosphate-buffered saline (PBS) or $10 \%$ fetal bovine serum (FBS) solution was placed into a dialysis tube (20 kD MWCO, Spectrum Laboratories, Rancho Dominguez, CA, USA). The dialysis tube was placed in $6 \mathrm{~mL}$ of PBS or $10 \%$ FBS and gently stirred with a magnetic stirrer in an incubator at $37^{\circ} \mathrm{C}$. $0.5 \mathrm{~mL}$ of aliquots was taken at fixed time intervals from the medium outside the dialysis tube, and the medium was immediately replenished with the same amount of PBS or 10\% FBS. Quantitation of CL released in PBS was measured by HPLC described above, while CL released in $10 \%$ FBS was quantified by UPLC/MS/MS analysis. The release ratio (\% released) was calculated by (2), where $M_{\text {released }}$ is the mass of the released CL and $M_{\text {encapsulated }}$ is the mass of CL encapsulated within the micelles.

$$
\% \text { released }=\frac{M_{\text {released }}}{M_{\text {encapsulated }}} \times 100 \% \text {. }
$$

2.8. Pharmacokinetics Study. Male Sprague-Dawley rats (250-300 g) were anesthetized with zoletil $(25 \mathrm{mg} / \mathrm{kg}, \mathrm{im})$ and xylazine $(10 \mathrm{mg} / \mathrm{kg}$, im), and body temperature was maintained at $37^{\circ} \mathrm{C}$ with a heating pad. Polyethylene catheters were inserted into the jugular vein for blood sampling. Rats were injected intravenously with an equivalent CL dose $(22 \mathrm{mg} / \mathrm{kg}$ ) of P-MCL, MCL, and free CL (the CL powder was dissolved in $0.9 \% \mathrm{NaCl}$-injectable solution by adding a few drops of $85 \%$ phosphoric acid). $500 \mu \mathrm{L}$ of blood samples was collected into $\mathrm{K}_{2}$ EDTA tubes (BD, Franklin Lakes, NJ, USA) via the jugular vein at postinjection time points $(0.05$, $0.25,0.5,1,2,4,8$, and 24 hours). Plasma samples were obtained after centrifugation at $4000 \mathrm{rpm}$ for 15 minutes and were stored at $-80^{\circ} \mathrm{C}$ until analysis.



FIGURE 2: Stability of P-MCLs with different PLGA/PEG-PE feeding ratios in $10 \%$ FBS. The hydrodynamic diameter changes of P-MCLs were monitored by DLS analysis.

2.9. Plasma Sample Extraction. Plasma sample extraction was performed as previously reported [25]. Briefly, $10 \mu \mathrm{L}$ of ROX solution $(6 \mu \mathrm{g} / \mathrm{mL}$ in $50 \%$ acetonitrile) as the internal standard (IS), $60 \mu \mathrm{L}$ of acetonitrile, and $50 \mu \mathrm{L}$ of plasma sample were mixed. After centrifugation at $3500 \mathrm{rpm}$ for $10 \mathrm{~min}$, $15 \mu \mathrm{L}$ of supernatant was transferred to a 96-well SPE plate conditioned with methanol and water. The extracted samples were eluted with $150 \mu \mathrm{L}$ of acetonitrile and evaporated using a SpeedVac concentrator (Refrigerated Vapor Trap RVT4104, Thermo Fisher Scientific, Waltham, MA, USA). The residue was reconstituted in $150 \mu \mathrm{L}$ of $50 \%$ acetonitrile, and $5 \mu \mathrm{L}$ of aliquot was injected into the UPLC/ $\mathrm{MS} / \mathrm{MS}$ system. The peak area ratio of CL to IS was calculated to determine the CL concentration.

2.10. UPLC/MS/MS Condition. UPLC/MS/MS condition was followed as previously reported [25]. Briefly, chromatographic separation was performed on a Waters ACQUITY UPLC system (Milford, MA, USA) with a Waters $\mathrm{BEH} \mathrm{C}_{18}$ column $(50 \mathrm{~mm} \times 2.1 \mathrm{~mm}, 1.7 \mu \mathrm{m}$ id, Milford, MA, USA) maintained at $40^{\circ} \mathrm{C}$. The mobile phase consisted of solvent A (acetonitrile) and solvent B (50 mM ammonium acetate) with a flow rate of $0.3 \mathrm{~mL} / \mathrm{min}$. The initial mobile phase composition was $50 \%$ solvent $\mathrm{A}$, changed linearly to $10 \%$ solvent A (0-1.5 min) and held for $0.2 \mathrm{~min}(1.5-1.7 \mathrm{~min})$, followed by a return to the initial condition within $0.1 \mathrm{~min}(1.7-1.8 \mathrm{~min})$, and kept for $0.2 \mathrm{~min}(1.8-2.0 \mathrm{~min})$ for the column equilibrium. The UPLC system was connected to an ACQUITY TQD tandem mass spectrometer (Waters, Manchester, UK) as an electrospray ionization interface monitoring in positive ionization mode. Quantitation was performed using multiple reaction monitoring to study precursor $\rightarrow$ product ion transitions of the 


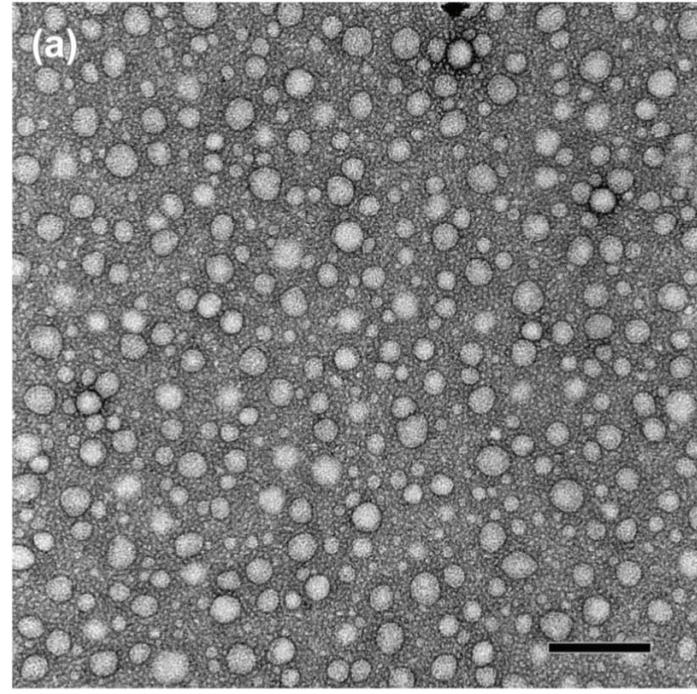

(a)

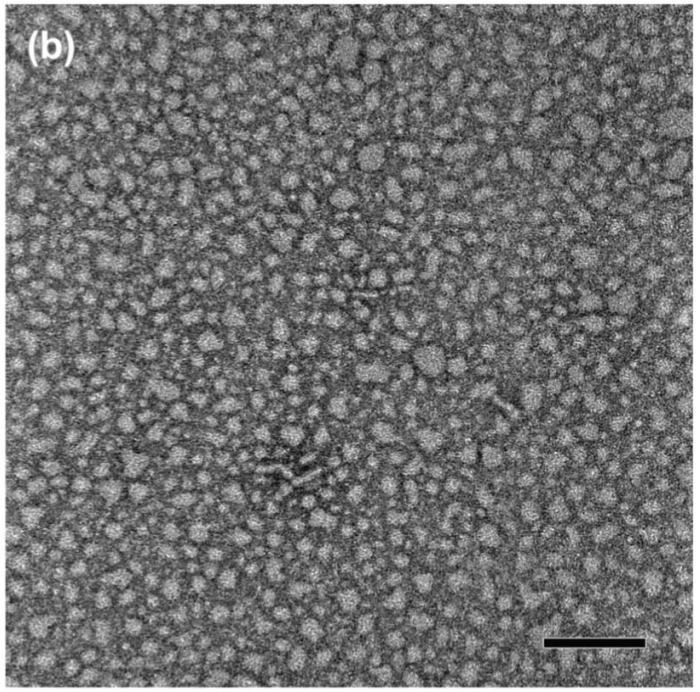

(b)

Figure 3: TEM images of (a) P-MCL and (b) MCL negatively stained by uranyl acetate (scale bars: $100 \mathrm{~nm}$ ).

analyte and internal standard of $\mathrm{m} / z 748.77 \rightarrow 83.04$ and $837.84 \rightarrow 116.05$ for CL and ROX, respectively.

2.11. Pharmacokinetics Analysis. The plasma concentrationversus-time data of CL from the mean of three rats was analysed to determine the pharmacokinetic parameters using a noncompartmental model in WinNonlin Professional version 5.2 (Pharsight, Mountain View, CA, USA). The area under the curve (AUC), half-life $\left(t_{1 / 2}\right)$, clearance, mean residence time (MRT), and volume distribution $\left(V_{\mathrm{d}}\right)$ were evaluated. The management of animal experiment and animal care and use of NHRI has been accredited by the AAALAC International.

\section{Results and Discussion}

\subsection{Results}

3.1.1. Optimization of PLGA Incorporation. To understand how much PLGA should be incorporated to stabilize micelle structure, P-MCLs with different PLGA/PEG-PE feeding ratios were prepared and incubated in 10\% FBS. Their size changes were monitored by DLS (Figure 2) to examine the stability of P-MCLs over time. When the PLGA/PEG-PE feeding ratio was 0.5 , the size of P-MCL remained almost the same around $75 \mathrm{~nm}$ from 0 to 65 hours at $37^{\circ} \mathrm{C}$. In contrast, with the 0.25 feeding ratio, the size of P-MCL increased dramatically from 80 to $275 \mathrm{~nm}$ after 17 hours and exhibited an obvious cloudy appearance. The similar size increment (size change from 80 to $380 \mathrm{~nm}$ after 41 hours) and cloudy appearance were observed while the feeding ratio was 1 . Therefore, the optimal PLGA/PEG-PE feeding ratios was determined as 0.5 .

3.1.2. Characterization of P-MCL and MCL. P-MCL and MCL were prepared by a single emulsification-solvent evaporation method and were observed under TEM with negative stain. P-MCL has a spherical shape (Figure 3(a)), and MCL
TABle 1: Physicochemical properties of P-MCL and MCL.

\begin{tabular}{lcccc}
\hline $\begin{array}{l}\text { Sample } \\
n=4\end{array}$ & $\begin{array}{c}\text { Mean } \\
\text { diameter } \\
(\mathrm{nm})\end{array}$ & $\begin{array}{c}\text { Zeta } \\
\text { potential } \\
(\mathrm{mV})\end{array}$ & $\begin{array}{c}\text { Loading } \\
\text { content } \\
(\%)\end{array}$ & $\begin{array}{c}\text { Entrapment } \\
\text { efficiency } \\
(\%)\end{array}$ \\
\hline P-MCL & $35.1 \pm 14.3$ & $-34.2 \pm 0.8$ & $15.7 \pm 0.9$ & $45.7 \pm 5.2$ \\
MCL & $32.0 \pm 9.1$ & $-37.2 \pm 1.3$ & $15.1 \pm 0.7$ & $57.9 \pm 5.5$ \\
\hline
\end{tabular}

has a fluffy irregular shape (Figure 3(b)). Table 1 summarizes the general physicochemical properties of P-MCL and MCL. They have similar average diameters (below $40 \mathrm{~nm}$ ), high CL loading contents (about 15\%), and negative surface charges (around $-35 \mathrm{mV}$ ).

3.1.3. In Vitro Release Profiles of P-MCL and MCL. The in vitro $\mathrm{CL}$ release profiles from $\mathrm{P}-\mathrm{MCL}$ and $\mathrm{MCL}$ in different release media are given in Figure 4. In PBS (Figure 4(a)), during the initial 6 hours, $30 \%$ and $50 \%$ of the loaded CL were released from P-MCL and MCL, respectively. After 6 hours, the CL was released in a much slower manner from both P-MCL and MCL. After incubation for 48 hours, the CL released from P-MCL and MCL amounted to 51.3\% and $81.1 \%$, respectively. When the media was switched to $10 \%$ FBS (Figure 4(b)), there was no obvious burst release of CL from P-MCL and MCL. At 6 hours, only 7\% of the loaded CL was released from P-MCL, while CL released from MCL was about $12 \%$. The CL was gradually released from both P-MCL and MCL during the observation period for 72 hours. Overall, P-MCL had a slower CL release rate than MCL in both PBS and 10\% FBS solution.

3.1.4. Pharmacokinetics. Pharmacokinetic profiles of three types of CL administration (P-MCL, MCL, and free CL) were obtained in male Sprague-Dawley rats. Figure 5 shows the CL concentrations in plasma. CL concentrations from all the three administrations decreased rapidly within the first hour and then decreased in a one-exponential-decay 


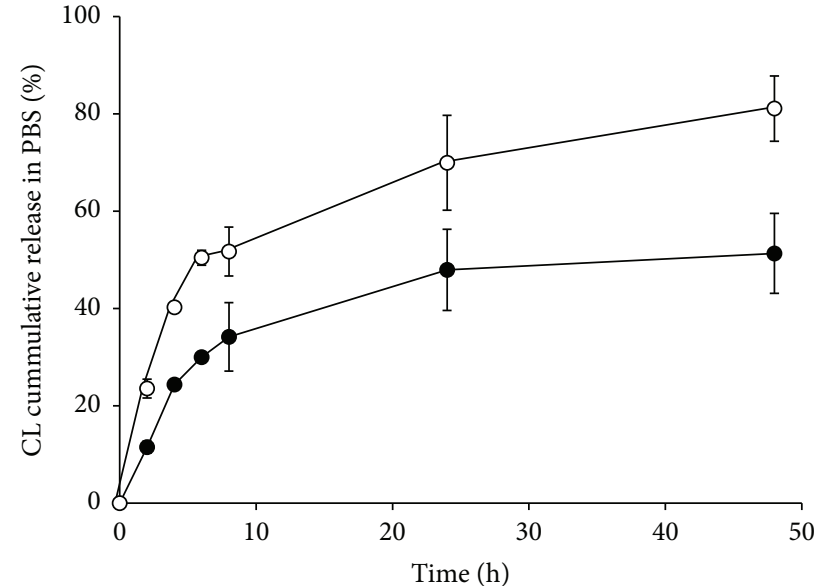

$-\mathrm{P}-\mathrm{MCL}$
$-\mathrm{O}-\mathrm{MCL}$

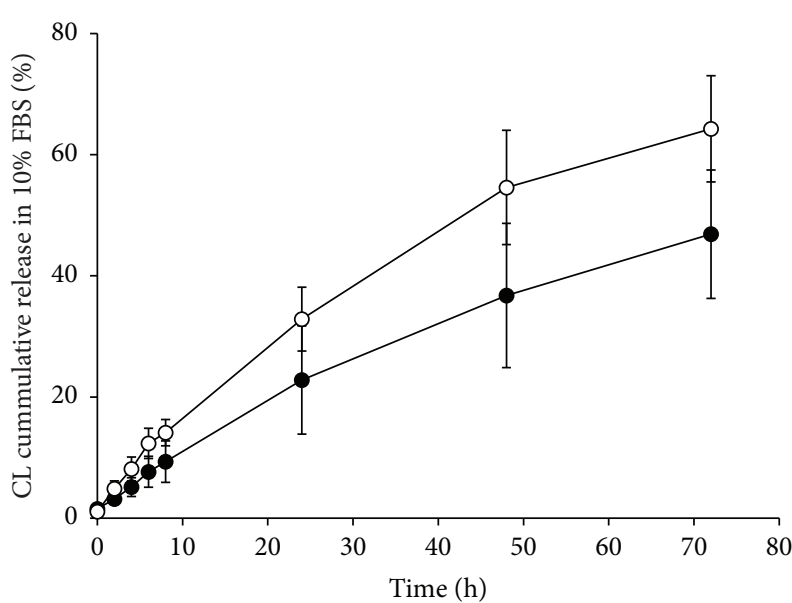

$-\mathrm{P}-\mathrm{MCL}$
$-\mathrm{O}-\mathrm{MCL}$

(a)

(b)

FIGURE 4: In vitro release profiles of P-MCL and MCL in (a) PBS and (b) $10 \%$ FBS solution $(n=3)$.

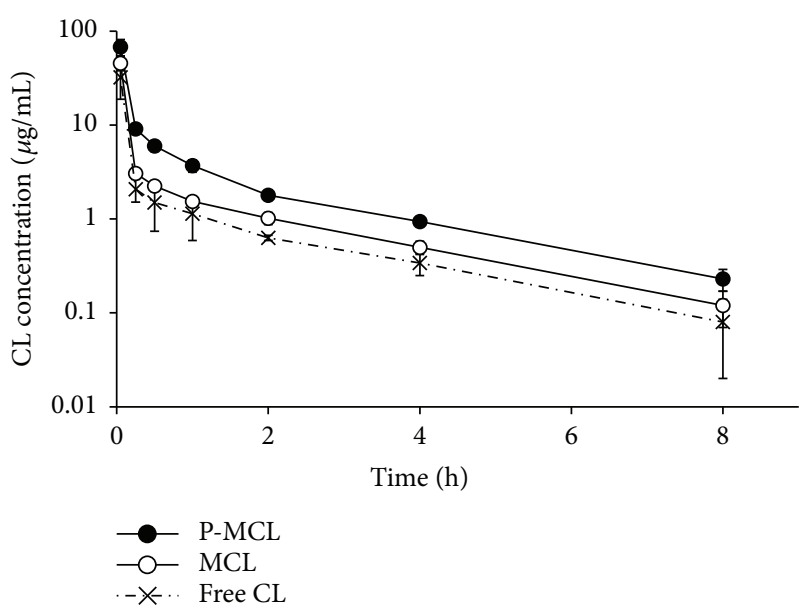

FIGURE 5: Mean plasma concentration of CL-time curves after iv administration of P-MCL, MCL, and free CL $(22 \mathrm{mg} / \mathrm{kg}, n=3)$ in rats.

pattern. P-MCL had the highest CL concentration in plasma at every time point $(0.05,0.25,0.5,1,2,4,8$, and 24 hours). The pharmacokinetic parameters were analysed and are presented in Table 2. The AUC value was in the order of P-MCL $(24.41 \pm 1.51 \mathrm{~h} \cdot \mu \mathrm{g} / \mathrm{mL})>\mathrm{MCL} \quad(13.92 \pm 2.09 \mathrm{~h} \cdot \mu \mathrm{g} / \mathrm{mL})>$ free CL $(9.66 \pm 3.21 \mathrm{~h} \cdot \mu \mathrm{g} / \mathrm{mL})$, showing that the blood CL concentrations of P-MCL and MCL were 2.5-fold and 1.4-fold higher, respectively, than that of free CL. The clearance and $V_{\mathrm{d}}$ were in the order of free CL $>$ MCL $>$ P-MCL. The clearance rates of P-MCL and MCL were 2.7-fold and 1.5-fold lower, respectively, than that of free CL. The AUC and clearance of P-MCL were 1.8-fold higher and 1.8-fold slower, respectively, than those of MCL.

3.2. Discussion. The stability issue of premature drug release from micelles resulting from the dynamic exchange of
TABLE 2: Pharmacokinetic parameters of P-MCL, MCL, and free CL.

\begin{tabular}{lcccc}
\hline & Unit & P-MCL & MCL & Free CL \\
\hline Dosage & $\mathrm{mg} / \mathrm{kg}$ & 22 & 22 & 22 \\
$t_{1 / 2}$ & $\mathrm{~h}$ & $2.04 \pm 0.27$ & $1.91 \pm 0.27$ & $1.87 \pm 0.26$ \\
AUC & $\mathrm{h} \cdot \mu \mathrm{g} / \mathrm{mL}$ & $24.41 \pm 1.51$ & $13.92 \pm 2.09$ & $9.66 \pm 3.21$ \\
Clearance & $\mathrm{L} / \mathrm{h} / \mathrm{kg}$ & $0.88 \pm 0.05$ & $1.57 \pm 0.27$ & $2.37 \pm 0.65$ \\
MRT & $\mathrm{h}$ & $1.04 \pm 0.14$ & $0.94 \pm 0.07$ & $0.92 \pm 0.12$ \\
$V_{\mathrm{d}}$ & $\mathrm{L} / \mathrm{kg}$ & $2.60 \pm 0.46$ & $4.28 \pm 0.39$ & $6.54 \pm 2.51$ \\
\hline
\end{tabular}

unimers or the adsorption of serum proteins is one of the obstacles for developing an effective micellar nanocarrier in pharmaceutical applications [26, 27]. The premature drug leakage could decrease drug therapeutic efficiency and lead to undesirable toxicity to other tissues. Therefore, it is important to enhance the stability of micelles for preventing premature drug release in the physiological environment.

In the present study, we incorporated hydrophobic PLGA into PEG-PE micelles to enhance the micelle stability. While the PLGA/PEG-PE feeding ratio is $0.5, \mathrm{P}-\mathrm{MCL}$ shows the best stability property in $10 \%$ FBS with no size increment over time, suggesting that no aggregation occurred (Figure 2). With this optimal amount of PLGA incorporation, P-MCL has a slower CL release rate than MCL has in both PBS and 10\% FBS solutions (Figure 3), indicating its better micelle stability. In the pharmacokinetic study, PMCL showed a higher AUC and a lower clearance than did MCL (Table 2), which means that P-MCL was cleared more slowly and supplied more available CL in vivo. Since free $\mathrm{CL}$ was cleared very fast, the lower clearance of P-MCL may be contributed by the less drug leakage due to the better protection from the higher micelle stability. The hydrophobic PLGA copolymer here plays a crucial role for increasing the hydrophobic interaction to the PE carbon chains of the PEG-PE unimers. 
Despite of the decrease in the critical micellar concentration by adjusting the chemical composition of unimers $[28,29]$, micelle stability can be generally enhanced by chemical modification or by physical interaction approaches [30]. Among chemical modification approaches, the hydrophilic or hydrophobic segment of micelle unimers can be cross-linked to improve micelle integrity [23]. The crosslinked micelles had higher AUC than non-cross-linked micelles had in murine blood [31, 32]. Using the crosslinking strategy may reduce degradation or collapse of micelles, but whether the cross-linked products are still biocompatible and biodegradable is still a concern [33]. Meanwhile, drugs may not be releasable when they are entrapped in a nondegradable vehicle (only the released drugs are essential for the therapeutic efficacy) [27].

Instead of chemical modification, physical mixing of hydrophobic materials within the micelles is a convenient approach to enhance micelle stability. For example, a hydrophobic vegetable oil was introduced into pluronic micelles which exhibited less degradation than did original pluronic micelles upon dilution [34]. Physical mixing methods provide a simple way to improve micelle stability via increased noncovalent forces like hydrophobic interaction, aromatic $\pi-\pi$ interaction, hydrogen bonding, and ionic interaction between unimers, drugs, and mixing materials [30]. Moreover, these kinds of noncovalent interactions may provide enough force to stabilize micelles, yet drugs can be released controllably from moderate entrapment.

Here we selected the physical mixing strategy to improve micelle stability by PLGA incorporation. Because of the convenience of physical mixing, we can further change the hydrophobic blending materials like different molecule weights or different L/G ratios of the PLGA or can adjust PEG lengths by switching PEG-lipid materials for optimized drug encapsulation or micelle stability.

\section{Conclusions}

Micelles composed of PEG lipids have emerged as promising drug delivery vehicles for hydrophobic drugs to enhance the drug availability or delivery efficiency. However, PEG-lipid micelles have a stability issue, in that unimers are in a dynamic equilibrium between micelles and bulk solution, resulting in an uncontrollable drug release or leakage. When a hydrophobic polymer such as PLGA was incorporated into the micellar core, the carbon chains of PEG-PE unimers could be interlaced by PLGA which reduce the dynamic exchange of PEG-PE unimers and thereby reduce the burst release and release rate of entrapped drugs. Moreover, micelles with improved stability by PLGA interlacing exhibited lower clearance, higher area under plasma concentration curve, and lower volume distribution than did nonstabilized micelles in a murine pharmacokinetic study, providing more advantages in controllable micellar delivery systems.

\section{Conflicts of Interest}

The authors report no declarations of interest.

\section{Acknowledgments}

This research was supported by intramural grants from the National Health Research Institutes (BN-106-PP-23 and BN-106-PP-25), Taiwan.

\section{References}

[1] O. C. Farokhzad and R. Langer, "Impact of nanotechnology on drug delivery," ACS Nano, vol. 3, no. 1, pp. 16-20, 2009.

[2] J. Shi, A. R. Votruba, O. C. Farokhzad, and R. Langer, "Nanotechnology in drug delivery and tissue engineering: from discovery to applications," Nano Letters, vol. 10, no. 9, pp. 3223-3230, 2010.

[3] A. Z. Wilczewska, K. Niemirowicz, K. H. Markiewicz, and H. Car, "Nanoparticles as drug delivery systems," Pharmacological Reports, vol. 64, no. 5, pp. 1020-1037, 2012.

[4] R. A. Petros and J. M. DeSimone, "Strategies in the design of nanoparticles for therapeutic applications," Nature Reviews Drug Discovery, vol. 9, no. 8, pp. 615-627, 2010.

[5] L. Wu, J. Zhang, and W. Watanabe, "Physical and chemical stability of drug nanoparticles," Advanced Drug Delivery Reviews, vol. 63, no. 6, pp. 456-469, 2011.

[6] F. Danhier, E. Ansorena, J. M. Silva, R. Coco, A. Le Breton, and V. Préat, "PLGA-based nanoparticles: an overview of biomedical applications," Journal of Controlled Release, vol. 161, no. 2, pp. 505-522, 2012.

[7] M. L. Immordino, F. Dosio, and L. Cattel, "Stealth liposomes: review of the basic science, rationale, and clinical applications, existing and potential," International Journal of Nanomedicine, vol. 1, no. 3, pp. 297-315, 2006.

[8] T. M. Allen and P. R. Cullis, "Liposomal drug delivery systems: from concept to clinical applications," Advanced Drug Delivery Reviews, vol. 65, no. 1, pp. 36-48, 2013.

[9] V. P. Torchilin, "Lipid-core micelles for targeted drug delivery," Current Drug Delivery, vol. 2, no. 4, pp. 319-327, 2005.

[10] V. P. Torchilin, "Micellar nanocarriers: pharmaceutical perspectives," Pharmaceutical Research, vol. 24, no. 1, pp. 1-16, 2007.

[11] K. K. Gill, A. Kaddoumi, and S. Nazzal, "PEG-lipid micelles as drug carriers: physiochemical attributes, formulation principles and biological implication," Journal of Drug Targeting, vol. 23, no. 3, pp. 222-231, 2015.

[12] K. S. Soppimath, T. M. Aminabhavi, A. R. Kulkarni, and W. E. Rudzinski, "Biodegradable polymeric nanoparticles as drug delivery devices," Journal of Controlled Release, vol. 70, no. 1-2, pp. 1-20, 2001.

[13] A. Kumari, S. K. Yadav, and S. C. Yadav, "Biodegradable polymeric nanoparticles based drug delivery systems," Colloids and Surfaces B: Biointerfaces, vol. 75, no. 1, pp. 1-18, 2010.

[14] N. Jawahar and S. N. Meyyanathan, "Polymeric nanoparticles for drug delivery and targeting: a comprehensive review," International Journal of Health \& Allied Sciences, vol. 1, no. 4, pp. 217-223, 2012.

[15] D. Owens III and N. Peppas, "Opsonization, biodistribution, and pharmacokinetics of polymeric nanoparticles," International Journal of Pharmaceutics, vol. 307, no. 1, pp. 93-102, 2006.

[16] A. N. Lukyanov, Z. Gao, L. Mazzola, and V. P. Torchilin, "Polyethylene glycol-diacyllipid micelles demonstrate increased 
accumulation in subcutaneous tumors in mice," Pharmaceutical Research, vol. 19, no. 10, pp. 1424-1429, 2002.

[17] Z. Gao, A. N. Lukyanov, A. Singhal, and V. P. Torchilin, "Diacyllipid-polymer micelles as nanocarriers for poorly soluble anticancer drugs," Nano Letters, vol. 2, no. 9, pp. 979-982, 2002.

[18] A. N. Lukyanov, W. C. Hartner, and V. P. Torchilin, "Increased accumulation of PEG-PE micelles in the area of experimental myocardial infarction in rabbits," Journal of Controlled Release, vol. 94, no. 1, pp. 187-193, 2004.

[19] Z. Gao, A. N. Lukyanov, A. R. Chakilam, and V. P. Torchilin, "PEG-PE/phosphatidylcholine mixed immunomicelles specifically deliver encapsulated Taxol to tumor cells of different origin and promote their efficient killing," Journal of Drug Targeting, vol. 11, no. 2, pp. 87-92, 2003.

[20] L. Mu, T. A. Elbayoumi, and V. P. Torchilin, "Mixed micelles made of poly(ethylene glycol)-phosphatidylethanolamine conjugate and $\mathrm{D}$ - $\alpha$-tocopheryl polyethylene glycol 1000 succinate as pharmaceutical nanocarriers for camptothecin," International Journal of Pharmaceutics, vol. 306, no. 1-2, pp. 142-149, 2005.

[21] A. Patist, S. G. Oh, R. Leung, and D. O. Shah, "Kinetics of micellization: its significance to technological processes," Colloids and Surfaces A: Physicochemical and Engineering Aspects, vol. 176, no. 1, pp. 3-16, 2001.

[22] T. Nicolai, O. Colombani, and C. Chassenieux, "Dynamic polymeric micelles versus frozen nanoparticles formed by block copolymers," Soft Matter, vol. 6, no. 14, pp. 3111-3118, 2010.

[23] R. Trivedi and U. B. Kompella, "Nanomicellar formulations for sustained drug delivery: strategies and underlying principles," Nanomedicine, vol. 5, no. 3, pp. 485-505, 2010.

[24] C.-H. Chu, Y.-C. Wang, H.-Y. Huang, L.-C. Wu, and C.S. Yang, "Ultrafine PEG-coated poly(lactic-co-glycolic acid) nanoparticles formulated by hydrophobic surfactant-assisted one-pot synthesis for biomedical applications," Nanotechnology, vol. 22, no. 18, article 185601, 2011.

[25] Y.-J. Wang, Y.-T. Wu, J.-Y. Lin et al., "Rapid quantitative analysis of clarithromycin in rat plasma by UPLC-MS/MS after intravenous injection of the clarithromycin-loaded ultrafine PLGA nanoparticles," Journal of Chromatography $B$, vol. 895-896, pp. 178-181, 2012.

[26] H. M. Burt, X. Zhang, P. Toleikis, L. Embree, and W. L. Hunter, "Development of copolymers of poly(D,L-lactide) and methoxypolyethylene glycol as micellar carriers of paclitaxel," Colloids and Surfaces B: Biointerfaces, vol. 16, no. 1-4, pp. 161-171, 1999.

[27] Y. Shi, T. Lammers, G. Storm, and W. E. Hennink, "Physicochemical strategies to enhance stability and drug retention of polymeric micelles for tumor-targeted drug delivery," Macromolecular Bioscience, vol. 17, no. 1, article 1600160, 2017.

[28] R.-Z. Xiao, Z.-W. Zeng, G.-L. Zhou, J. J. Wang, F. Z. Li, and A. M. Wang, "Recent advances in PEG-PLA block copolymer nanoparticles," International Journal of Nanomedicine, vol. 5, pp. 1057-1065, 2010.

[29] K. Zhang, X. Tang, J. Zhang et al., "PEG-PLGA copolymers: their structure and structure-influenced drug delivery applications," Journal of Controlled Release, vol. 183, pp. 77-86, 2014.

[30] W. Zhou, C. Li, Z. Wang, W. Zhang, and J. Liu, "Factors affecting the stability of drug-loaded polymeric micelles and strategies for improvement," Journal of Nanoparticle Research, vol. 18, no. 9, article 275, 2016.

[31] C. J. Rijcken, C. J. Snel, R. M. Schiffelers, C. F. van Nostrum, and W. E. Hennink, "Hydrolysable core-crosslinked thermosensitive polymeric micelles: synthesis, characterisation and in vivo studies," Biomaterials, vol. 28, no. 36, pp. 5581-5593, 2007.

[32] Y. Li, K. Xiao, J. Luo et al., "Well-defined, reversible disulfide cross-linked micelles for on-demand paclitaxel delivery," Biomaterials, vol. 32, no. 27, pp. 6633-6645, 2011.

[33] X. Ke, V. W. L. Ng, R. J. Ono et al., "Role of non-covalent and covalent interactions in cargo loading capacity and stability of polymeric micelles," Journal of Controlled Release, vol. 193, no. 9-26, pp. 9-26, 2014.

[34] N. Rapoport, "Stabilization and activation of Pluronic micelles for tumor-targeted drug delivery," Colloids and Surfaces B: Biointerfaces, vol. 16, no. 1-4, pp. 93-111, 1999. 


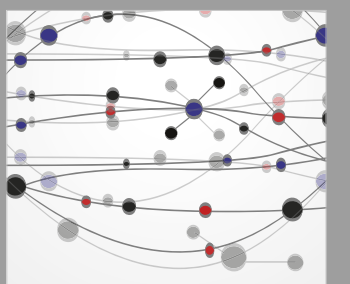

The Scientific World Journal
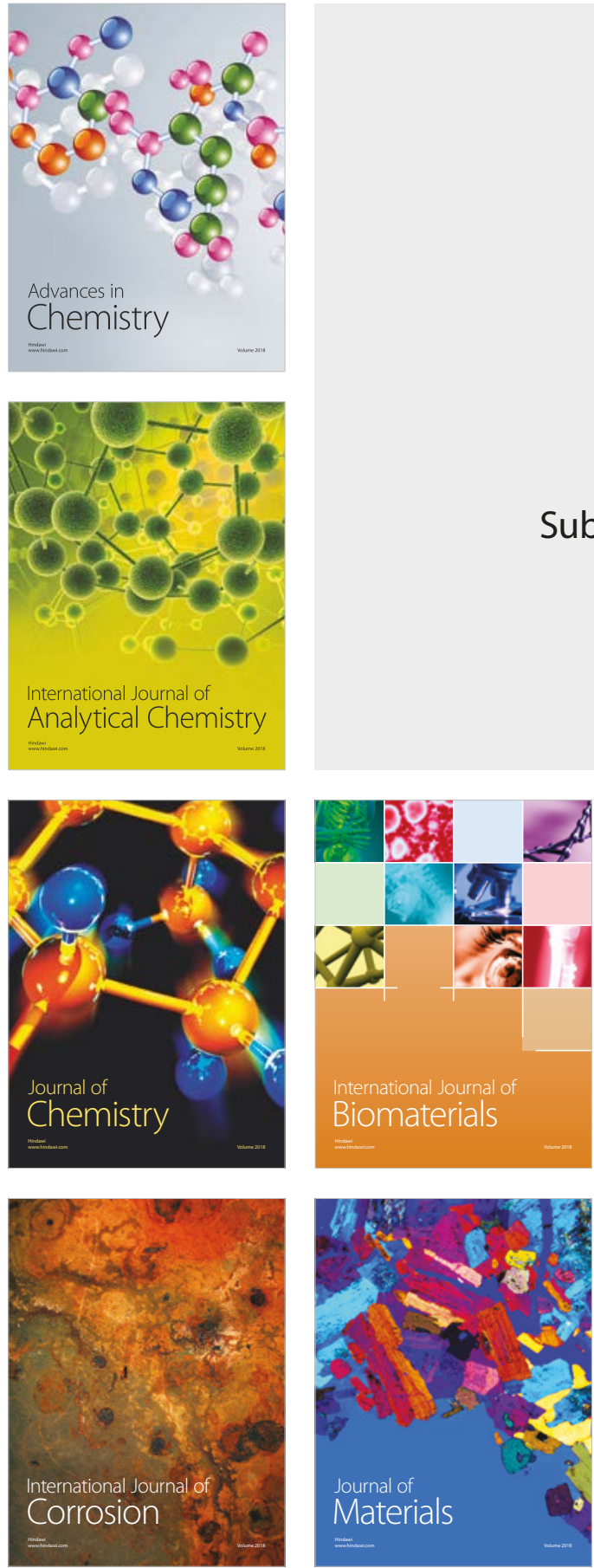



Journal of

Applied Chemistry


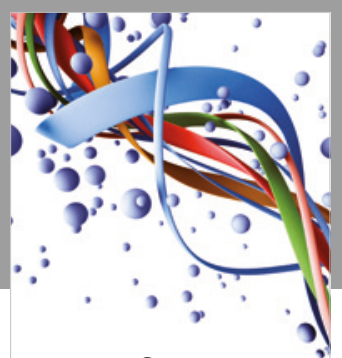

Scientifica

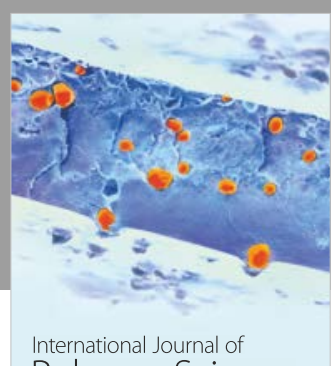

Polymer Science

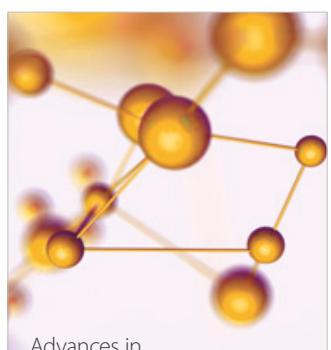

Physical Chemistry
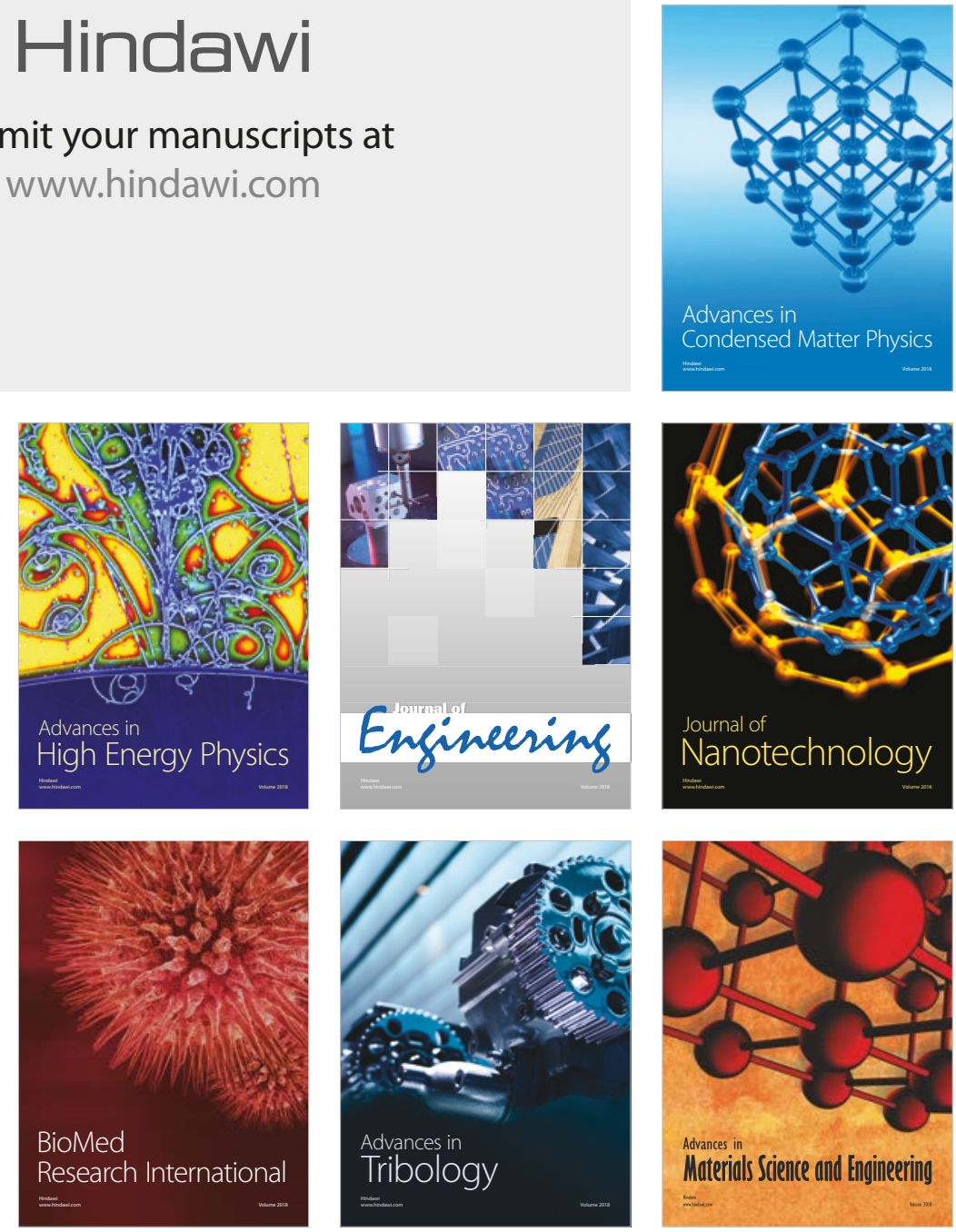